\title{
THE CULTIVATION OF HUMAN GRANULOSA CELLS
}

 \\ Jaroslav Mokry $\tilde{z}^{2}$
}

University of Pardubice, Faculty of Chemical-Technology, Czech Republic: Department of Biological and Biochemical Science $^{1}$; Charles University in Prague, Faculty of Medicine in Hradec Králové, Czech Republic: Department of Histology and Embryology ${ }^{2}$; Sigma-Aldrich, Prague, Czech Republic ${ }^{3}$; Charles University in Prague, $1^{\text {st }}$ Medical Faculty and General Teaching Hospital in Prague, Czech Republic: Department of Obstetrics and Gynecology, Assisted Reproduction Center ${ }^{4}$

\begin{abstract}
Summary: The major functions of granulosa cells (GCs) include the production of steroids, as well as a myriad of growth factors to interact with the oocyte during its development within the ovarian follicle. Also FSH stimulates GCs to convert androgens (coming from the thecal cells) to estradiol by aromatase. However, after ovulation the GCs produce progesterone that may maintain a potential pregnancy. Experiments with human GCs are mainly focused on the purification of GCs from ovarian follicular fluid followed by FACS analysis or short-term cultivation. The aim of our study was to cultivate GCs for a long period, to characterize their morphology and phenotype. Moreover, we have cultivated GCs under gonadotropin stimulation in order to simulate different pathological mechanisms during folliculogenesis (e.g. ovarian hyperstimulation syndrome). GCs were harvested from women undergoing in vitro fertilization. Complex oocyte-cumulus oophorus was dissociated by hyaluronidase. The best condition for transport of GCs was optimized as short transport in follicular fluid at $37{ }^{\circ} \mathrm{C}$. GCs expansion medium consisted of DMEM/F12, 2 \% FCS, ascorbic acid, dexamethasone, L-glutamine, gentamycine, penicillin, streptomycin and growth factors (EGF, bFGF). GCs transported in follicular fluid and cultivated in $2 \%$ FCS containing DMEM/F12 medium supplemented with follicular fluid presented increased adhesion, proliferation, viability and decreased doubling time. Cell viability was $92 \%$ and mean cell doubling time was 52 hrs. We have optimized transport and cultivation protocols for long-term cultivation of GCs.
\end{abstract}

Key words: Human granulosa cells; Cultivation protocol; Proliferation potential

\section{Introduction}

Granulosa cells (GCs) are somatic cells found closely associated with the developing oocyte in the ovarian follicle. Follicular cells form a single flattened layer around the mammalian oocyte in the primordial ovarian follicle. During a development of the follicle (folliculogenesis) they proliferate and start to be called the granulosa cells (in multilaminar primary follicle). After the formation of follicular antrum (in the secondary or antral follicul) they form stratified granulosa layer. In the mature (graafian) follicle, they also surround the oocyte in a form of corona radiata, which is a part of cumulus oophorus. Human granulosa-lutein cells (lutein cells) are today usually harvested during in vitro fertilization (IVF) from the pre-ovulatory follicles of hormonally stimulated women. These cells have been challenged with luteinizing hormone ( $\mathrm{LH}$ ) and human chorionic gonadotropin (hCG), which may be regarded as a signal converting GCs to lutein cells. This event has been described as the transformation of a protein-secreting cell to a steroid- secreting cell, which opposes proliferation and induces differentiation (11)

GCs undergo major morphological changes that correlate very well with modulation of their steroidogenic capacity. The process includes changes in intercellular contacts and communication, in cell membrane receptors, and in the development and organization of organelles associated with steroidogenesis (i.e. mitochondria, smooth endoplasmic reticulum, lipid droplets and lysosomes). This rearrangement and down-regulation of actin and actin-binding proteins is characteristic for GCs as well as for other cells like Leydig and adrenal cells which exhibit high levels of steroidogenesis (1). Once the follicle has accumulated few thousands GCs, a fluid-filled cavity (the antrum) becomes apparent. Three different populations of GCs have been suggested, based on the content of enzymes and secretion of proteins, steroids and acids.

As a consequence of antrum formation, three populations of GCs become evident: (i) mural GCs connected to the basement membrane; (ii) antral population comprises 
the cells closest to the antral cavity; and (iii) cumulus cells, surrounding the oocyte $(13,22,17)$.

During last days of follicular maturation, there is an increase in cell size and general metabolic activity (protein and RNA content) and decrease in cell proliferation in GCs population (9). Most of the autocrine or paracrine factors, such as steroids, gonadal peptides, and growth factors, which modulate GCs differentiation, exert their biological effects in a paradoxical manner: in early stages of follicular development, they are believed to be mitogenic while in later stages, they enhance GCs differentiation and luteinization in a coordinated manner with gonadotropin-cAMP-generated signals (3).

GCs are stimulated to grow, differentiate and luteinize by endocrine, paracrine and autocrine factors (2). In spite of having several hundred thousands of follicles in the mammalian ovary being in primordial and primary stages of development, only very few of them in each cycle become fully mature, while others are eliminated by atresia, a process that exhibits both the biochemical and the morphological features of programmed cell death (18). Moreover, in each reproductive cycle, a new corpus luteum is formed while the old one degenerate in a process called luteolysis, which is by nature an apoptotic process (14).

The production of a mature oocyte is a complex process during which germ cells and somatic cells maintain a close association. Despite its apparent simplicity, the growth of the mammalian oocyte depends on the ordered series of maturation and differentiation steps, in both the germ and the somatic cells. It has been shown that the expression of germ cell functions during follicle growth can be either cellautonomous or dependent on GCs differentiation. Moreover, it becomes evident that the oocyte itself can modulate and influence GCs differentiation and normal follicular development (7).

In fact, oocytes influence steroidogenesis, maintance of estradiol production and inhibition of progesterone production (21), induce GCs proliferation (20) and suppress expression of LH receptor mRNA in GCs (10). Moreover, oocytes stimulate secretion of hyaluronic acid that accumulates between cumulus cells as a viscoelastic extracellular matrix (16).

The extracellular matrix (ECM) between follicular cells and in the follicular fluid, is believed to regulate GCs function during follicular development. It was proved that shape of GCs, their survival, proliferation and steroidogenesis can be modulated in vitro by pure extracellular matrix components in a specific and coordinated manner. It is suggested that, in vivo, fibronectin and laminin would sustain follicular development by enhancing the survival and proliferation of GCs, whereas type I collagen might participate in the maintenance of estradiol secretion (12). The addition of laminin to the culture medium or laminin coating of culture wells stimulated progesterone secretion by rat GCs and promoted the enlargement of steroidogenic cells. Fibronectincoated, but not collagen I-coated, wells similarly promoted progesterone secretion. These results suggest that a cell adhesion receptor (an integrin) and also laminin and fibronectin, the major glycoprotein components of ECM, play important roles in the differentiation of GCs to lutein cells (4).

During in vitro fertilization (IVF), aspirates may be mixed with blood and thus contaminated with leukocytes and erythrocytes. A high rate of leukocyte contamination has been described in primary cultures of lutein cells enriched by the density gradients Ficoll ${ }^{\mathrm{TM}}$ and Percoll ${ }^{\mathrm{TM}}$ (5, 6 ). These density gradient media are not totally inert and have a potential to elicit the cellular activation in leukocytes during separation procedure (15).

Especially, when the role of cytokines in reproduction is to be investigated, both serum-free and leukocyte-free cultures are an advantage. Serum is an undefined substance containing multiple factors that might influence cell function. The content of polypeptides, hormones, growth factors and cytokines or binding proteins soluble receptors is undefined. However, without serum, normal cell function may be endangered and experiments must be limited in time.

The aim of our study was to cultivate GCs in low serum containing media and to characterize their morphology in vitro. Moreover, we had to optimize transport and cultivation conditions for long-term cultivation.

\section{Material and Methods}

GCs isolation. GCs were obtained from 4 consecutive patients with their informed consent according to the Guidelines of Assisted Reproduction Center, Prague. GCs were harvested from human preovulatory follicles in the course of oocyte retrieval for IVF. The mean age of patients was 33.8 yrs (range from 31-37 yrs). Patients were stimulated by 10.000 IU Pregnyl (hCG, N.V. Organon Oss, Netherlands) $36 \mathrm{hrs}$ prior oocytes collection.

At an average 7 oocytes were harvested together with GCs from each patient. GCs were isolated from corona radiata and a part of cumulus oophorus. This suspension was enzymatically treated using 80 IU Hyaluronidase (FertiPro N.V.) to release oocytes from GCs. Oocytes were evacuated with glass capillary and afterwards, inactivation medium was added into the GCs suspension. GCs inactivation medium consisted of DMEM (Dulbecco's Modified Eagle's Medium)/F12 (Sigma-Aldrich, USA), 10 \% fetal calf serum (FCS) (PAA, Sweden), 200 mM L-glutamine (Gibco, Scotland), $10 \mathrm{mg} / \mathrm{ml}$ gentamycine (Gibco, Scotland), $2.5 \mathrm{mg} / 1$ amphotericin (Sigma-Aldrich, USA), 10.000 units/ml penicillin and $10.000 \mu \mathrm{g} / \mathrm{ml}$ streptomycin (Gibco, Scotland). GCs suspension was centrifuged at $357 \mathrm{G}$ for $5 \mathrm{~min}$ at $20^{\circ} \mathrm{C}$.

Transport protocol optimisation. The pellet containing GCs was resuspended either in transport medium [compounded of DMEM/F12, 2 \% FCS, L-glutamine, gentamycine, amphotericin, penicillin and streptomycin] or in transport buffer [PBS (Gibco, Scotland), gentamycine, amphotericin, penicillin and streptomycin]. 
Subsequent conditions for transport were tested:

a) transport buffer $\left(4^{\circ} \mathrm{C}\right)$,

b) transport medium $\left(4^{\circ} \mathrm{C}\right)$,

c) transport buffer $\left(37^{\circ} \mathrm{C}\right)$,

d) transport medium $\left(37^{\circ} \mathrm{C}\right)$,

e) follicular fluid $\left(37^{\circ} \mathrm{C}\right)$,

f) some GCs samples were also cryopreserved and stored in liquid nitrogen prior the transport.

Cultivation protocol optimization. In our laboratory GCs were centrifugated ( $357 \mathrm{~g}$ for $5 \mathrm{~min}$ ), then resuspended in cultivation medium and seeded. GCs expansion medium consisted of DMEM/F12. 2 or $10 \%$ FCS, $10 \mathrm{mg} / \mathrm{ml}$ ascorbic acid (Sigma-Aldrich, USA), $0.05 \mu \mathrm{M}$ dexamethasone (Sigma-Aldrich, USA), $200 \mathrm{mM}$ L-glutamine, $10 \mathrm{mg} / \mathrm{ml}$ gentamycine, 10.000 units $/ \mathrm{ml}$ penicillin and $10.000 \mu \mathrm{g} / \mathrm{ml}$ streptomycin. Moreover we added $2 \mu \mathrm{l} / \mathrm{ml}$ epidermal growth factor (EGF) and $5 \mu \mathrm{l} / \mathrm{ml}$ basic fibroblast growth factor (bFGF) (PeproTech, USA). Cultivation medium was changed every day. Cells were cultivated at $37{ }^{\circ} \mathrm{C}$ under aerobic conditions $\left(5 \% \mathrm{CO}_{2}\right)$.

GCs were cultivated:

a) on untreated plastic (Petri-dishes, TPP, Switzerland) in $10 \%$ FCS-containing DMEM/F12 medium,

b) on untreated plastic (Petri-dishes, TPP, Switzerland) in $2 \%$ FCS containing DMEM/F12 medium,

c) on untreated plastic of 6-well plate (Corstar, USA) in $2 \%$ FCS containing DMEM/F12 medium supplemented with $2 \mathrm{ml}$ follicular fluid.

Once adherent cells were more than $50 \%$ confluent. They were detached with $0.25 \%$ trypsin-EDTA for $10 \mathrm{~min}$ (Gibco, Scotland), counted using Z2 counter or cell viability analyser Vi-Cell XR 2.03 (both Beckman Coulter, USA). Microscope Nicon Eclipse TE300 and digital camera Olympus DP12 were used to make pictures.

\section{Results}

Transport protocol optimization. GCs were harvested from women underwent IVF, complex oocyte-cumulus oophorus was dissociated by hyaluronidase. Following inactivation, GCs were transported to our laboratory. Transport of GCs in transport buffer and transport medium at $4{ }^{\circ} \mathrm{C}$ decreased their adhesion and proliferation capability. Some samples were also cryopreserved and stored in liquid nitrogen prior the transport. Nevertheless most of the GCs transported in liquid nitrogen lost their adhesion and proliferation.
Transport in medium at $37{ }^{\circ} \mathrm{C}$ was necessary for maintenance of average cell viability $44 \%$ (Tab. 1). The best conditions for transport of GCs were short transport (max. 2 hrs) in follicular fluid (FF) at $37{ }^{\circ} \mathrm{C}$. These conditions supported adhesion and proliferation potential of GCs (Figs. $1-4)$.

Cultivation protocol optimization. Our initial experiment with GCs harvested from human preovulatory follicles done immediately following transport showed average cell count 125.150 cells $/ \mathrm{ml}$ and cell viability was only $44 \%$ (Tab. 1). Proliferation potential of these GCs increased following the addition of mitogens, EGF and bFGF.

Subsequent experiments were designed according to our initial results. GCs were cultivated on untreated plastic in $2 \%$ and $10 \%$ FCS-containing DMEM/F12 expansion medium supplemented with growth factors EGF and bFGF. Using this cultivation protocol, GCs adhered to surface at $3 \mathrm{hrs}$ after inoculation. GCs cultivated in $2 \%$ FCS containing medium were heterogenous population of cells with multiple processes when seeded. After 5 days in culture, cells adopted variable shapes with clearly visible cytoskeleton (Fig. 1). Cell doubling time for GCs cultivated in $2 \%$ FCS was 1.736 hrs. During the whole in vitro cultivation we have also measured GCs count summarized in Tab. 2.

GCs cultivated in $10 \%$ FCS containing medium formed also heterogenous population of cells. Forty eight hours after inoculation, cells appeared to be widespread, and multiple contacts between the cells were noted. GCs cultivated in $10 \%$ FCS assumed more flatten and multiform morphology. Their cell doubling time was $2.210 \mathrm{hrs}$. GCs cultivated in $10 \%$ FCS degenerated faster (day 18) than GCs cultivated in $2 \%$ FCS containing medium (day 28). This degenerative process was characterized by increased cell diameter and vacuoles appeared in the cytoplasm (Fig. 2).

Tab. 1: Cell viability, cell count and other parameters of initial experiment GCs.

\begin{tabular}{|c|c|c|}
\hline Patient & No. 1 & No. 2 \\
\hline Oocytes count & 5 & 8 \\
\hline Transport temp. & $37^{\circ} \mathrm{C}$ & $37^{\circ} \mathrm{C}$ \\
\hline GCs count & 113.900 & 136.400 \\
\hline GCs viability & $47 \%$ & $41 \%$ \\
\hline Cultivation media & $\begin{array}{c}\text { DMEM/F12, } \\
10 \% \mathrm{FCS}, \\
\text { glutamine }\end{array}$ & $\begin{array}{c}\text { DMEM/F12 } \\
\text { DCS, glutamine, } \\
\text { EGF, bFGF }\end{array}$ \\
\hline
\end{tabular}

Tab. 2: GCs total count during the whole in vitro cultivation.

\begin{tabular}{|c|c|c|c|c|c|}
\hline $\begin{array}{c}\text { Days in } \\
\text { culture }\end{array}$ & $\begin{array}{c}\mathbf{2 \%} \\
\text { FCS }\end{array}$ & $\begin{array}{c}\mathbf{1 0} \% \\
\text { FCS }\end{array}$ & $\begin{array}{c}\text { Days in } \\
\text { culture }\end{array}$ & $\begin{array}{c}\mathbf{2} \% \text { FCS + } \\
\text { transport in FF }\end{array}$ & $\begin{array}{c}\mathbf{2} \text { \% FCS + } \\
\text { supplemented with FF }\end{array}$ \\
\hline 6 & $242.900 \mathrm{GCs} / \mathrm{ml}$ & $107.500 \mathrm{GCs} / \mathrm{ml}$ & 7 & $113.120 \mathrm{GCs} / \mathrm{ml}$ & $136.320 \mathrm{GCs} / \mathrm{ml}$ \\
\hline 20 & $316.250 \mathrm{GCs} / \mathrm{ml}$ & $156.600 \mathrm{GCs} / \mathrm{ml}$ & 11 & $161.800 \mathrm{GCs} / \mathrm{ml}$ & $143.500 \mathrm{GCs} / \mathrm{ml}$ \\
\hline 28 & $201.400 \mathrm{GCs} / \mathrm{ml}$ & 0 & 21 & $383.200 \mathrm{GCs} / \mathrm{ml}$ & $1.240 .000 \mathrm{GCs} / \mathrm{ml}$ \\
\hline- & - & - & 25 & $563.100 \mathrm{GCs} / \mathrm{ml}$ & $4.330 .000 \mathrm{GCs} / \mathrm{ml}$ \\
\hline Doubling time & $\mathbf{1 . 7 3 6} \mathbf{h r s}$ & $\mathbf{2 . 2 1 0} \mathbf{h r s}$ & - & $\mathbf{1 7 9} \mathbf{h r s}$ & $\mathbf{5 2} \mathbf{h r s}$ \\
\hline
\end{tabular}




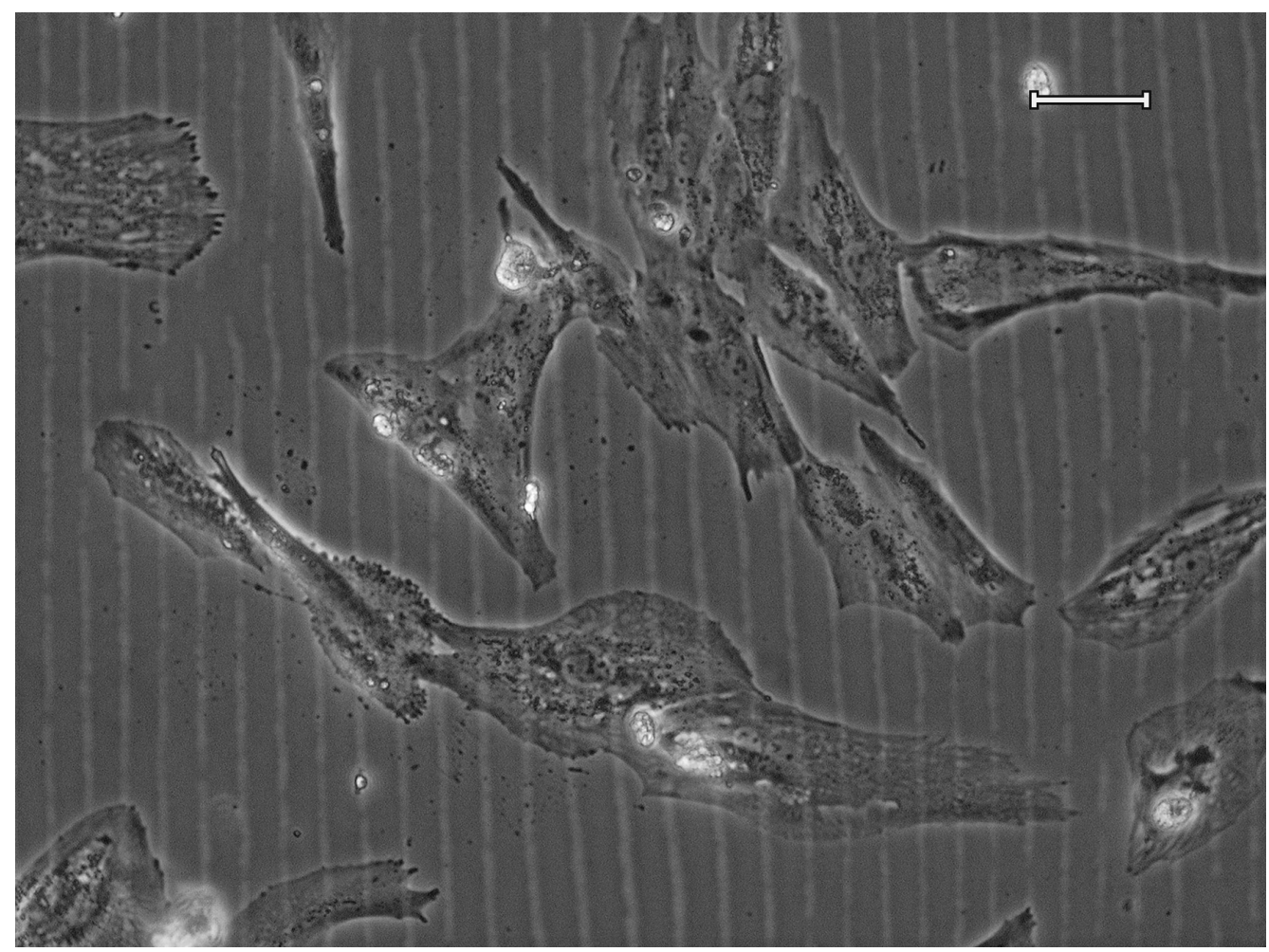

Fig. 1: Typical morphology of human GCs cultivated in $2 \%$ FCS medium on a plastic surface (day 18) and transported in a transport medium, bar $15 \mu \mathrm{m}$.

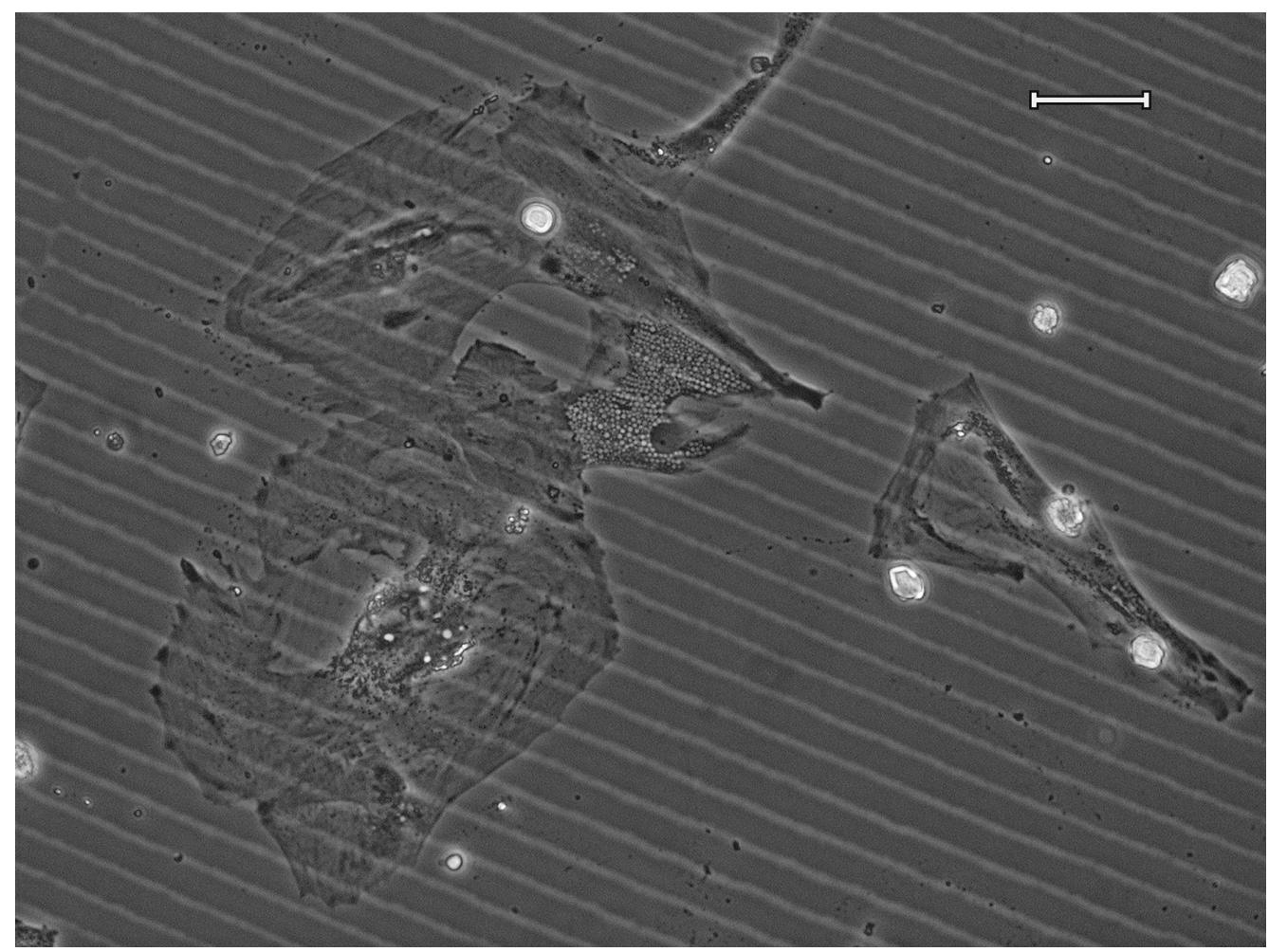

Fig. 2: Culture of human GCs undergoing degeneration process, was cultivated in $10 \%$ FCS medium on a plastic surface (day 18) and transported in a transport medium, bar $15 \mu \mathrm{m}$. 




Fig. 3: Human GCs transported in follicular fluid and cultivated in $2 \%$ FCS medium on a plastic surface (day 18), bar $15 \mu \mathrm{m}$.

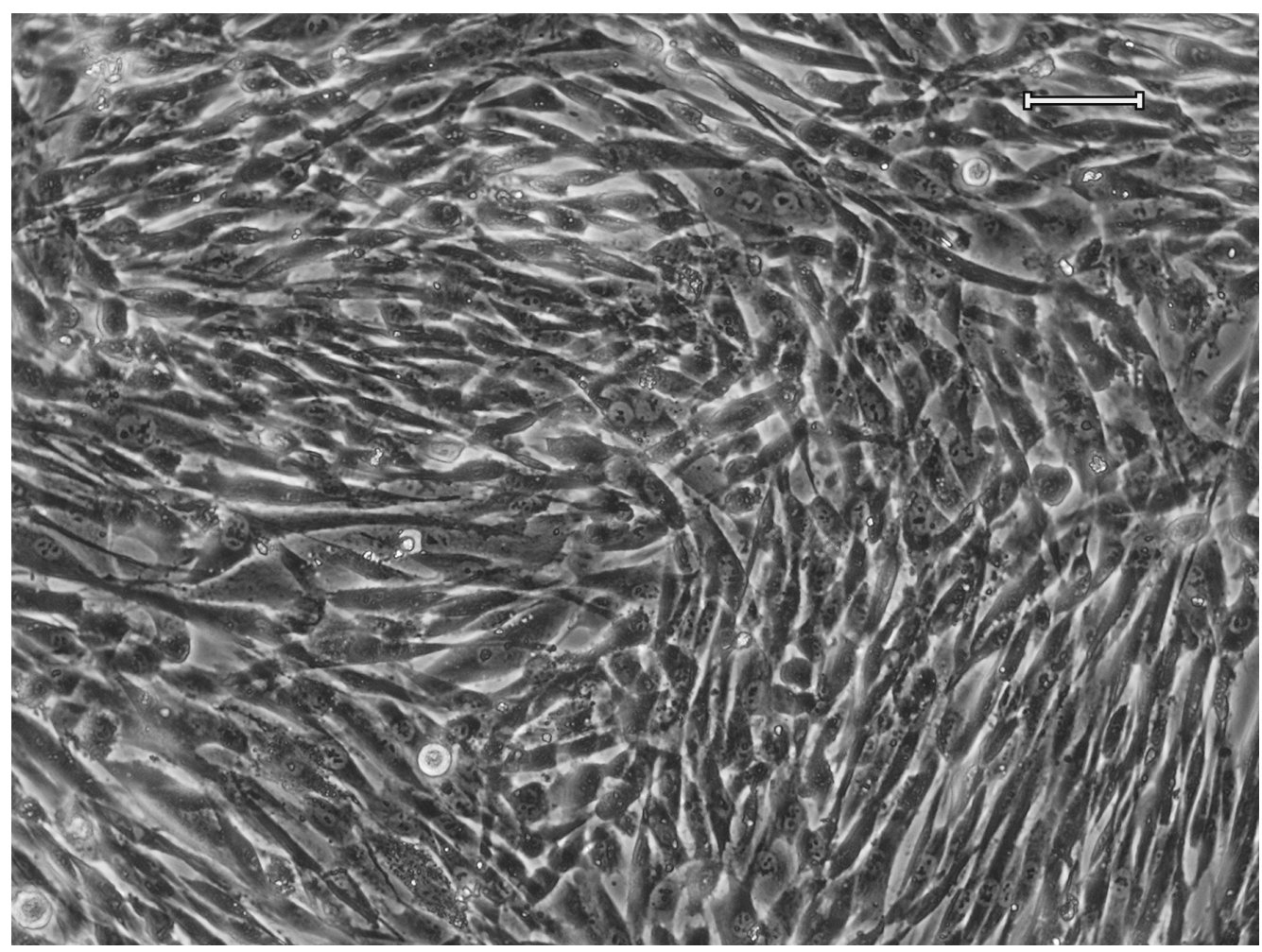

Fig. 4: Spindle-shaped human GCs which were transported in follicular fluid and cultivated in $2 \%$ FCS medium supplemented with follicular fluid on a plastic surface (day 18), bar $15 \mu \mathrm{m}$. 


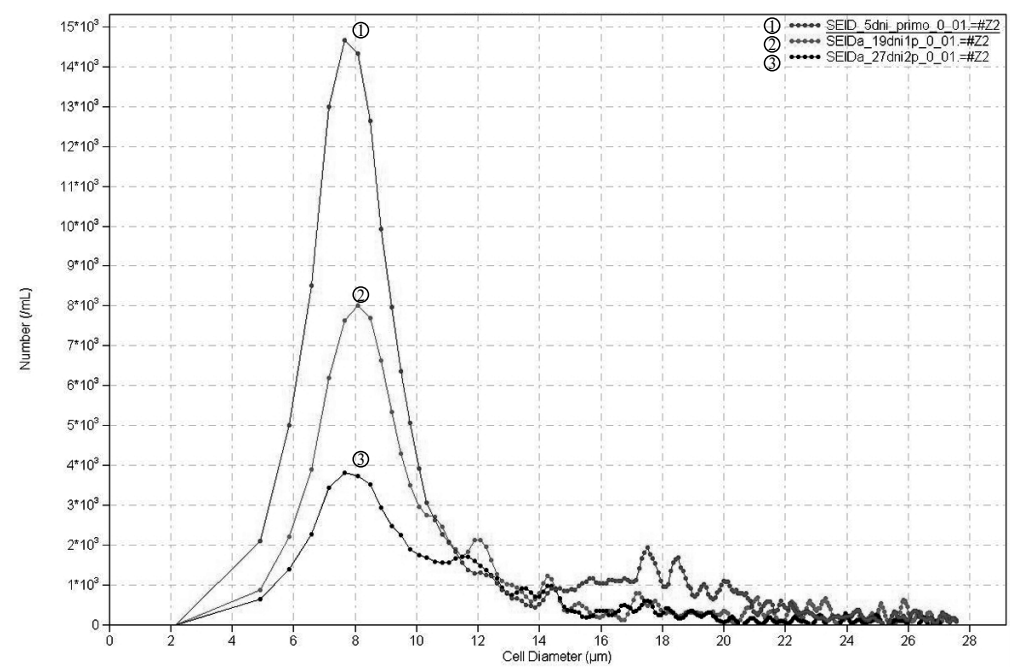

Graph 1: Comparison of GCs cultures cultivated in $2 \%$ FCS medium (at days 5, 19, 20)



Graph 2: Comparison of GCs cultivated in $10 \%$ FCS medium (at days 5 and 19).

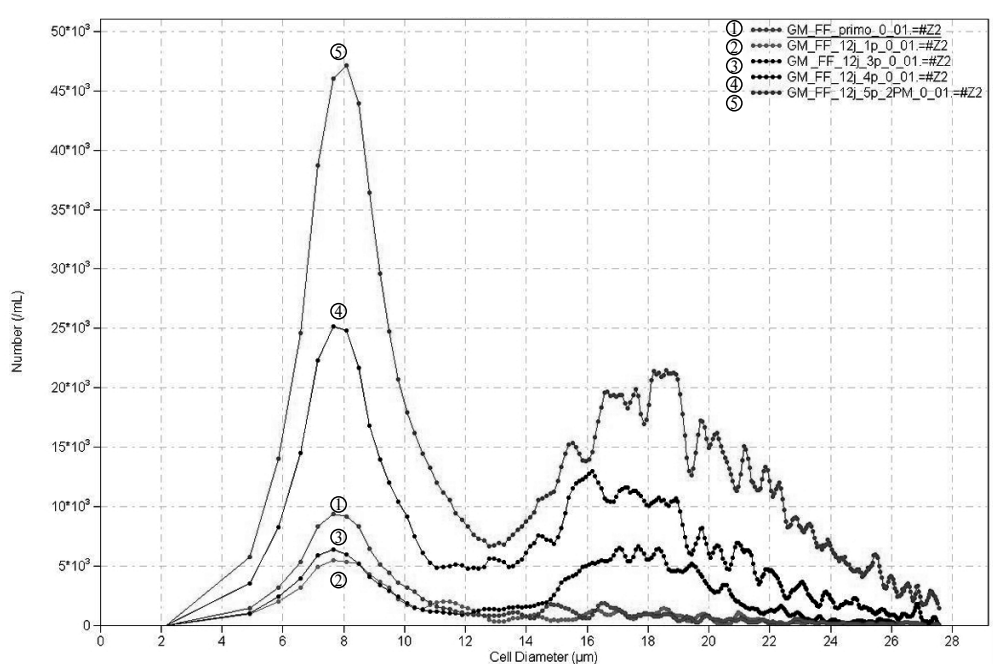

Graph 3: Comparison of GCs cultivated in $2 \%$ FCS medium supplemented with FF, transported in FF (at days 7, 11, 14, 18, 25). 
On the contrary, when FF was used for transport, GCs had the spherical shape when seeded and grew faster (Fig. 3). However, cell doubling time for GCs transported in FF was expressively lower $179 \mathrm{hrs}$ compared with GCs transported in transport medium (Tab. 2). The highest cell viability $92 \%$ and the lowest doubling time $52 \mathrm{hrs}$ were obtained when GCs were transported in FF and cultivated in $2 \%$ FCS containing DMEM/F12 medium supplemented with FF following inoculation. These GCs revealed higher adhesion capability, proliferation potential and cell viability, and significantly lower doubling time (Tab. 2). GCs assumed spindle-shaped morphology when we added FF in medium (Fig. 4). Experiment was finished after 7 passages (day 32).

During the whole in vitro expansion process we have analyzed GC diameter that remained stable between all passages of GCs cultivated either in 2 or $10 \%$ FCS (Graph $1,2)$. The average cell diameter in predominant GCs population ranged between 7.6 and $8.1 \mu \mathrm{m}$. GCs cultures cultivated in $10 \%$ FCS medium contained GCs with larger cell diameter (Graph 2).

GCs cultivated in $2 \%$ FCS medium supplemented with FF and transported in FF presented two subsequent cell populations. During the whole in vitro expansion process, the proportion of GCs population has changed. At the beginning of cultivation process, cell population of diameter 5-12 $\mu \mathrm{m}$ has dominated. Later on, cell population of diameter 14-26 $\mu \mathrm{m}$ has prevailed (Graph 3).

\section{Discussion}

GCs isolated from different species of animals were described by many authors. Experiments with human GCs were mainly focused on the purification of GCs from ovarian FF followed by FACS analysis or short-term cultivation with monitoring of progesterone production after the treatment with human gonadotropins.

In this report, we have shown that GCs can be transported and cultivated on the untreated plastic surface culture flasks without the stimulation by human gonadotropins. During the follicular phase of the menstrual cycle, elevations in serum FSH levels stimulate the GCs of small antral follicles to undergo a serie of mitotic divisions. FSH also induces the expression of $\mathrm{LH}$ receptors within the GCs. As a result of FSH stimulation, a preovulatory follicle develops with capability of ovulation in response to LH. The LH surge not only induces ovulation but also stimulates GCs of the preovulatory follicle to luteinize which results in transformation of the follicle in the corpus luteum. It appears that LH surge acts as a "switch", preventing cell proliferation and initiating luteinization, as evidenced by enhanced progesterone synthesis (8). It should be stressed that GCs were harvested closely before ovulation from corona radiata and a part of cumulus oophorus of hormonally stimulated women. GCs lose their high proliferative potential characteristic for early stages of follicular development during the growth of follicle. They start to differentiate and acquire the features characteristic for their steroidogenic function, witch culminates before ovulation (19).

We have described differences in morphology of GCs cultivated in $2 \%$ and $10 \%$ FCS containing expansion medium supplemented with growth factors, and influence of FF on cell proliferation potential. Serum (FCS) is an undefined substance with multiple factors that might influence cell function (11). This represents a disadvantage of our medium used for GCs. However, normal cell functions may be endangered in serum-free medium and experiment is therefore timelimited. GCs represented heterogenous population of cells with multiple cell processes when cultivated in $10 \%$ FCS containing medium. The cells appeared to be widespread, and multiple contacts between cells were noted $48 \mathrm{hrs}$ after inoculation. The generation of processes continued during the whole in vitro cultivation. GCs became more flattened and larger, and degenerated faster (at 18 days) unlike the GCs cultivated in $2 \%$ FCS containing medium (at 28 days). Cells were of spherical-shape with well-visible cytoskeleton. Less processes and connections between the cells were noted.

GCs were of spindle shape with many intercellular contacts, when FF was added in the medium. Cell doubling time for GCs transported in FF was expressively lower 179 hrs compared with doubling time 1.736-2.210 hrs of GCs transported in transport medium. The highest cell viability $92 \%$ and the lowest doubling time 52 hrs were obtained when GCs were transported in FF and cultivated in $2 \%$ FCS containing DMEM/F12 medium supplemented with FF following inoculation. These GCs revealed increased adhesion, proliferation, cell viability and decreased doubling time. The influence of FF on GCs proliferation have not described so far. Moreover FF is native source of growth factors, hormones, cytokines and other components for optimal development of GCs in vivo (18), therefore, we consider this transport protocol as advantageous. FF has formed membrane-like structure on the bottom of Petridish probably serving as a three-dimensional matrix important for cell communication and continuous proliferation. Our results showed that we were able to increase continuously cell count during 32 days of cultivation. Figenschau et al. (11) were unable to demonstrate an increase in cell count during 7 days of cultivation (GCs were cultivated in medium supplemented with either serum or SSR2TM synthetic serum replacement). Even addition of mitogens, EGF and bFGF, did not influence the cell proliferation in their experiment.

During the whole in vitro expansion process GCs population supplemented with FF changed its proportion (two different subsequent subpopulations were noted). At the beginning of cultivation process, the cells of diameter 5-12 $\mu \mathrm{m}$ have dominated. Later, during cultivation period, cell population of diameter $14-26 \mu \mathrm{m}$ started to predominate. It is likely that these two cell populations are in accordance with process which GCs undergo in vivo. Our findings document that small GCs with high proliferative potential are 
typical for early stages of the development of follicular cells, and after 4-5 passages, they start to differentiate and adopt features corresponding to steroidogenic function. The following study is necessary to characterize differences in phenotype of these two types of cells.

\section{Conclusions}

The aim of our study was to cultivate GCs in vitro and to characterize their morphology. Initially we had to optimize transport and cultivation protocols for long-term cultivation of GCs. We optimized low FCS containing expansion medium supplemented with EGF and bFGF. We have described differences in morphological characteristics of GCs cultivated in medium supplemented with follicular fluid and influence of FF on cell proliferation. GCs were very sensitive to any changes of environmental conditions (e.g. temperature, enzymatic treatment, rate of isolation, centrifugation speed etc.). In our further experiments, we would like to observe GCs of women who did not undergo in vitro fertilization and also we would like to cultivate GCs stimulated with gonadotropin to simulate different pathological mechanisms during folliculogenesis (e.g. ovarian hyperstimulation syndrome).

\section{Acknowledgements}

The authors would like to acknowledge whole the IVF group of Assisted Reproduction Center in Prague, Czech Republic for GCs harvesting, for their support and cooperation. We would like also to thank Mrs. H. Rückerová for her assistance in the tissue cultures laboratory and Ing. Milan Tománek, CSc., for his expertise and advice concerning GCs.

Work was supported by the research projects of the Ministry of Education of the Czech Republic, MSM 0021620820 and MSM0021627502, and by the IGA of the Ministry of Health of CR, NR/8932/3.

\section{References}

1. Amsterdam A, Plehn-Dujowich D, Suh BS. Structure-function relationship during differentiation of normal and oncogene transformed granulosa cells. Biol Reprod 1992;46:513-522.
2. Amsterdam A, Rotmensch S. Structure-function relationships during granulosa cell differentiation. Endocr Rev 1987;8:309-337.

3. Amsterdam A and Selvaraj NG. Control of differentiation, transformation, an apoptosis in granulosa cells by oncogenes, oncoviruses, and tumor suppressor genes. Endocr Rev 1997; 18:435-461.

4. Aten FR, Kolodecik TR, Behrman HR. A cell adhesion receptor antiserum abolishes, laminin and fibronectin glycoprotein components extracellular matrix promote, luteinization rat granulosa cells. Endocrinology 1995;136:1753-1758.

5. Beckmann MW, Polacek D, Seung L et al. Human ovarian granulosa cell culture determination of blood cell contamination and evaluation of possible purification steps. Fertil Steril 1991:56:881-887.

6. Best CL, Pudney J, Anderson DJ et al. Modulation of human granulosa cell steroid production in vitro by tumor necrosis factor $\alpha$ : implications of white blood cells in culture. Obstet Gynecol 1994;84:121-127.

7. Canipari R. Oocyte-granulosa cell interactions. Hum Reprod 2000; 6: 279-289.

8. Chaffkin LM, Luciano AA, Peluso JJ. The role of progesteron in regulating human granulosa cell proliferation and differentiation in vitro. J Clin Endocrinol Metab. 1993;76:696-700.

9. Dive C, Yoshida TM, Simpson DJ, Marrone BL. Flow cytometric analysis of steroidogenic organelles in differentiating granulosa cells. Biol Reprod 1992; 47:520-527.

10. Eppig JJ, Pendola FL, Wigglesworth K. Mouse oocytes suppress CAMP-induced expression of LH receptor mRNA by granulosa cells in vitro. Mol Reprod Dev 1998;49:327-332.

11. Figenschau Y, Sundsfjord JA, Yousef MI, Fuskevag OM, Sveinbjornsson B and Bertheussen K. A simplified serum-free method for preparation and cultivation of human granulosa-luteal cells. Hum Reprod 1997;12:523-531.

12. Huet C, Pisselet C, Mandon-Pepin B, Monget P, Monniaux D. Extracellular matrix regulates ovine granulosa cell survival, proliferation and steroidogenesis: relationships between cell shape and function. J Endocrinol 2001:169:347-360.

13. Inkster SE and Brodie AM. Expression of aromatase cytochrome P-450 in premenopausal and postmenopausal human ovaries: an immunocytochemical study. J Clin Endocrinol Metab 1991;73:717-726.

14. Jo T, Tomiyama T, Ohashi K, Saji F, Tanizawa O, Ozaki M et al. Apoptosis of cultured mouse luteal cells induced by tumor necrosis factor-alpha and interferon gamma. Anat Rec 1995;241:70-76.

15. Pickering SJ, Fleming TP, Braude PR et al. Are human spermatozoa separated on a Percoll density gradient safe for therapeutic use? Fertil Steril 1989;51: 1024-1029.

16. Salustri A, Yanagishita M, Hascall VC. Mouse oocytes regulate hyaluronic acid synthesis and mucification by FSH-stimulated cumulus cells. Dev Biol 1990; 138:26-32.

17. Salustri A, Yanagishita M, Underhill CB et al. Localization and synthesis of hyaluronic acid in the cumulus cells and mural granulosa cells of the preovulatory follicle. Dev Biol 1992;151:541-551

18. Tilly JL. Apoptosis and ovarian function. Rev Reprod 1996;1:162-172.20.

19. Tomanek M, Chronowska E, Kott T, et al. Telomerase activity in pig granulosa cells proliferating and differentiation in vitro. Anim Reprod Sci. 2008;104 284-298.

20. Vanderhyden BC, Telfer EE, Eppig JJ. Mouse oocytes promote proliferation of granulosa cells from preantral and antral follicles in vitro. Biol Reprod 1992; 46:1196-1204

21. Vanderhyden BC and Tonary AM. Differential regulation of progesterone and estradiol production by mouse cumulus and mural granulosa cells by A factor(s) secreted by the oocyte. Biol Reprod 1995;53:1243-1250.

22. Whitman GF, Boldt JP, Martinez JE, Pantazis CG. Flow cytometric analysis of induced human Graafian follicles. I. Demonstration and sorting of two luteinized cell populations. Fertil Steril 1991;56:259-265.

Submitted May 2008 Accepted September 2008

Corresponding author:

Mgr. Lenka Brůčková, Dept. of Histology and Embryology, Charles University in Prague, Faculty of Medicine in Hradec Králové, Šimkova 870, 50038 Hradec Králové, Czech Republic; e-mail: lbruckova@seznam.cz 